\title{
Californium versus cobalt brachytherapy combined with external-beam radiotherapy for IIB stage cervical cancer: long-term experience of a single institute
}

\author{
Ernestas Janulionis, MD, PhD', Prof. Konstantinas Povilas Valuckas, MD', Sarune Liukpetryte, MD', \\ Vitalija Samerdokiene, MD², Vydmantas Atkocius, PhD² \\ IRadiotherapy and Drug Center, ${ }^{2}$ Scientific Research Center, National Cancer Institute, Vilnius, Lithuania
}

\begin{abstract}
Purpose: The purpose of this paper was to observe and compare long-term curative effects and complications of FIGO stage IIB cervical cancer patients $(n=232)$ treated with high-dose-rate (HDR) californium $\left({ }^{252} \mathrm{Cf}\right)$ neutron or cobalt $\left({ }^{60} \mathrm{Co}\right)$ photon intracavitary brachytherapy (ICBT) combined with external-beam radiotherapy (EBRT).

Material and methods: The EBRT dose to the small pelvis was $50 \mathrm{~Gy}$ in both groups. The brachytherapy component of ${ }^{252} \mathrm{Cf}$ or ${ }^{60} \mathrm{Co}$ was added in the $3^{\text {rd }}$ week of EBRT, 5 fractions were performed once per week resulting in a total ICBT dose of $40 \mathrm{~Gy} / \mathrm{Gy}_{\text {eq }}$ (point A).

Results: Overall survival (OS) at 5, 10 and 15 years was $63.6 \%, 50.4 \%$ and $38.8 \%$ in the ${ }^{252} \mathrm{Cf}$ group and $62.2 \%, 50.5 \%$, $39.9 \%$, in the ${ }^{60} \mathrm{Co}$ group, respectively $(p=0.74)$. The percentage of tumour recurrence was statistically significantly lower in the ${ }^{252} \mathrm{Cf}$ group with $7.4 \%$ versus $17.1 \%$ in the ${ }^{60} \mathrm{Co}$ group $(p=0.02)$. Second primary cancers have developed similarly $9.1 \%$ and $8.1 \%$ cases for ${ }^{252} \mathrm{Cf}$ and ${ }^{60} \mathrm{Co}$ groups, respectively.

Conclusions: Our long-term retrospective study comparing ${ }^{252} \mathrm{Cf}$ and ${ }^{60} \mathrm{Co}$ isotopes with brachytherapy in combined treatment of FIGO IIB stage cervix carcinoma patients shows, that overall survival in the both groups are similar. However, the recurrence of tumour was significantly lower in the ${ }^{252} \mathrm{Cf}$ group. The incidence of second primary cancers was similar in both groups.
\end{abstract}

Key words: brachytherapy, ${ }^{252} \mathrm{Cf}$, cervical cancer, ${ }^{60} \mathrm{Co}$, neutron brachytherapy.

\section{Purpose}

Cervical cancer is still the leading cause of cancer deaths in women. Among women, it is the fourth leading cause of cancer worldwide by incidence and mortality in 2012. In Lithuania, its incidence is ranked in third place, and its mortality in sixth place [1].

A combination of intracavitary brachytherapy (ICBT) with external beam radiotherapy (EBRT) is used to treat advanced cervical cancer. Gamma radioactive nuclides such as ${ }^{60} \mathrm{Co},{ }^{137} \mathrm{Cs}$, and ${ }^{192} \mathrm{Ir}$ are commonly used in brachytherapy to treat cervical cancer patients.

Gamma rays provide low linear energy transfer radiation. ${ }^{252} \mathrm{Cf}$ as a neutron emitting radioactive isotope, produces high linear energy transfer (high-LET) radiation. High-LET radiation provides several radiobiological benefits: a relatively high biological effectiveness value (RBE), inhibition of cell damage repair, lower dependence of radiation sensitivity on the cell cycle, as well as a low oxygen enhancement ratio [2]. These advantages of highLET radiotherapy are most pronounced in the treatment of locally advanced tumors containing high proportions of hypoxic cells; in slowly growing tumors containing high proportions of cells that are in the radio resistant phases, as well as in tumors proliferating too fast to benefit from the conventional 6-week 30-fraction scheme [3].

In this paper, we retrospectively analyzed long-term data of 232 FIGO stage IIB patients treated from 1989 to 1999 with ${ }^{252} \mathrm{Cf}$ or ${ }^{60} \mathrm{Co}$ ICBT combined with EBRT at the National Cancer Institute, Vilnius, Lithuania. Our purpose was to observe and compare the long-term curative effects and complications.

\section{Material and methods \\ Patient population}

From 1989 to 1999, 232 stage IIB previously untreated patients with biopsy-proven cervical cancer were treated with HDR ${ }^{252} \mathrm{Cf}$ or ${ }^{60} \mathrm{Co}$ ICBT and EBRT at the National Cancer Institute, Vilnius: 121 patients were treated with neutron brachytherapy by ${ }^{252} \mathrm{Cf}$, and 111 with ${ }^{60} \mathrm{Co}$ ICBT. 
From October 1996 till April 1998, all patients were treated with neutron brachytherapy by ${ }^{252} \mathrm{Cf}$ combined with EBRT. The stage of disease was determined according to the FIGO classification on the basis of the following examinations: inspection, palpation, biopsy, cystoscopy, pyelography, abdominal ultrasonography, chest X-ray, and hematologic/biochemical tests of the blood. Patient characteristics are listed in Table 1. Eligibility criteria included histologically confirmed diagnosis. Information about radiotherapy was derived from hospital registry records and follow-up data records. The following data were also collected: age at initial diagnosis, pathological report, late radiation complications, hemoglobin level, body mass index, concurrent disease. During the follow-up period, the vital status of the subjects were determined using resident registration records available from the Death and Population Registers in our country, and causes of death were confirmed by the death certificate from the Archives Department under the government of the Lithuania.

The protocol for this study was approved by the Regional Biomedical Research Ethical Committee in Vilnius (No 158200-13-619-199, 2013-06-11).

\section{Treatment}

For both groups, EBRT was delivered by the ${ }^{60} \mathrm{Co}$ units AGAT-B and Rokus-M. The EBRT dose was delivered to the small pelvis through anteroposterior and posteroanterior portals $15-18$ by $15-19 \mathrm{~cm}$ at the skin surface. The applied total dose of 50 Gy (1.8-2.0 Gy per fraction) was divided to 16-18 Gy by full field, and the residual dose by split fields to the small pelvis. The central shielding of the split field was $4 \mathrm{~cm}$ in width and was applied in the third treatment week of EBRT after the 16-18 Gy.

The brachytherapy component of ${ }^{252} \mathrm{Cf}$ or ${ }^{60} \mathrm{Co}$ was added in the $3^{\text {rd }}$ week of EBRT. Intracavitary brachytherapy was administered once per week with a fraction dose of $8.0 \mathrm{~Gy} / \mathrm{Gy}_{\text {eq }}$ (point $\mathrm{A}$ ), and 5 fractions were performed resulting in a total ICBT dose of $40 \mathrm{~Gy} / \mathrm{Gy}_{\mathrm{eq}}$.
A USSR after loader (ANET-V) was used with three ${ }^{252} \mathrm{Cf}$ sources, which had an active length of $1.5 \times 9.0 \mathrm{~mm}$ : two static dwell positions at the lateral positions and one at the central applicator moving according proposed dwell time program (step $1 \mathrm{~cm}$ ). The ${ }^{252} \mathrm{Cf}$ source activities at the start was $1410.2 \mu \mathrm{g}$ for the central source and $360.4 \mu \mathrm{g}$ each for the lateral sources; and at the end of project $125.5 \mu \mathrm{g}$ and $32.1 \mu \mathrm{g}$, respectively. Irradiation time was in the interval from 10 minutes at the start to 100 minutes at the end of the project. The ICBT ${ }^{252} \mathrm{Cf}$ treatment irradiation dose-planning was performed by COSPO program (Russia) based on the Rjabukin [4] formula. The reference point was point $\mathrm{A}$. The treatment characteristics are shown in Table 2.

Follow ups were performed for each patient every 3 months in the first year after treatment and every 6 months thereafter.

\section{Statistical analysis}

Proportions were compared using the $\chi^{2}$ or Fisher test. The Kaplan-Meier method was used to estimate overall survival (OS) and disease-free survival (DFS). Differences between groups were calculated using the log-rank test. Univariate and multivariate Cox regression was performed to assess risk factors associated with mortality. $P$-value $<0.05$ was considered statistically significant. Analysis was performed using SPSS 21 software.

\section{Results}

The median follow-up for ${ }^{252} \mathrm{Cf}$ and ${ }^{60} \mathrm{Co}$ treated patients was 124.1 (interquartile range [IQR]: 24.9-206.5) and 118.3 (IQR: 34.4-177.4) months, respectively. The median age was 54 (IQR: $42-65$ ) years in the ${ }^{252} \mathrm{Cf}$ group and 50 (IQR: 42-61) years in the ${ }^{60}$ Co group.

The survival rates were calculated from the first treatment date to the last follow-up examination. The survival rates are shown in Figures 1 and 2. Overall survival at 5,

Table 1. Characteristics of IIB FIGO stage cervical cancer patients

\begin{tabular}{lcccc} 
& \multicolumn{2}{c}{ EBRT and ${ }^{252}$ Cf-ICBT } & \multicolumn{2}{c}{ EBRT and ${ }^{60}$ Co-ICBT } \\
\cline { 2 - 5 } & Patients & $\%$ & Patients & $\%$ \\
\hline Total patients & 121 & 100.0 & 111 & 100.0 \\
\hline Histopathology & & & 95 & 85.6 \\
\hline Squamous cell carcinoma & 113 & 93.4 & 9 & 8.1 \\
\hline Adenocarcinoma & 3 & 2.5 & 7 & 6.3 \\
\hline Others & 5 & 4.1 & 4 & 3.6 \\
\hline Histopathologic grade (G) & 1 & & 6 & 5.4 \\
\hline G1 (well differentiated) & 7 & 0.8 & 57 & 51.4 \\
\hline G2 (moderately differentiated) & 65 & 5.8 & 44 & 39.6
\end{tabular}

EBRT - external-beam radiotherapy, ${ }^{252} \mathrm{Cf}$ - californium-252, ICBT - intracavitary brachytherapy, ${ }^{60} \mathrm{Co}$ - cobalt-60 
Table 2. IIB FIGO stage cervical cancer patients treatment characteristics

\begin{tabular}{|c|c|c|}
\hline \multirow[t]{2}{*}{ Characteristics } & $\begin{array}{l}\text { EBRT and } \\
{ }^{252} \mathrm{Cf}-\mathrm{ICBT}\end{array}$ & $\begin{array}{l}\text { EBRT and } \\
{ }^{60} \mathrm{Co}-\mathrm{ICBT}\end{array}$ \\
\hline & Mean \pm SD & Mean \pm SD \\
\hline EBRT dose at point A (Gy) & $31.3 \pm 6.7$ & $34.3 \pm 6.2$ \\
\hline EBRT dose at point B (Gy) & $43.9 \pm 4.8$ & $44.8 \pm 5.5$ \\
\hline HDR brachytherapy dose (Gy) & $8.3 \pm 0.7$ & $8.0 \pm 0.2$ \\
\hline $\begin{array}{l}\text { HDR brachytherapy dose } \\
\text { at point A (Gy) }\end{array}$ & $43.6 \pm 5.6$ & $43.3 \pm 6.6$ \\
\hline $\begin{array}{l}\text { HDR brachytherapy dose } \\
\text { at point B (Gy) }\end{array}$ & $11.8 \pm 2.1$ & $11.6 \pm 4.3$ \\
\hline Cumulative point A dose & $74.9 \pm 7.2$ & $77.6 \pm 9.1$ \\
\hline Cumulative point $B$ dose & $55.7 \pm 4.2$ & $56.4 \pm 3.9$ \\
\hline Duration of EBRT (days) & $30.1 \pm 14.6$ & $28.8 \pm 12.2$ \\
\hline $\begin{array}{l}\text { Duration of HDR brachytherapy } \\
\text { (days) }\end{array}$ & $31.5 \pm 12.7$ & $30.5 \pm 10.8$ \\
\hline Duration of radiotherapy (days) & $53.7 \pm 32.6$ & $68.6 \pm 117.9$ \\
\hline Number of fractions & $5.3 \pm 0.7$ & $5.4 \pm 0.8$ \\
\hline
\end{tabular}

EBRT - external-beam radiotherapy, ${ }^{252} \mathrm{Cf}$ - californium-252, ICBT - intracavitary brachytherapy, ${ }^{60} \mathrm{Co}$ - cobalt-60, HDR - high-dose-rate

10 , and 15 years was $63.6 \%, 50.4 \%$ and $38.8 \%$ in the ${ }^{252} \mathrm{Cf}$ group, and $62.2 \%, 50.5 \%, 39.9 \%$ in the ${ }^{60} \mathrm{Co}$ group, respectively $(p=0.74)$.

In the ${ }^{252} \mathrm{Cf}$ group, the percentage of tumour recurrence was statistically significant lower than in the ${ }^{60} \mathrm{Co}$ group $(p=0.02)$. Also the percentage of distant metastases was

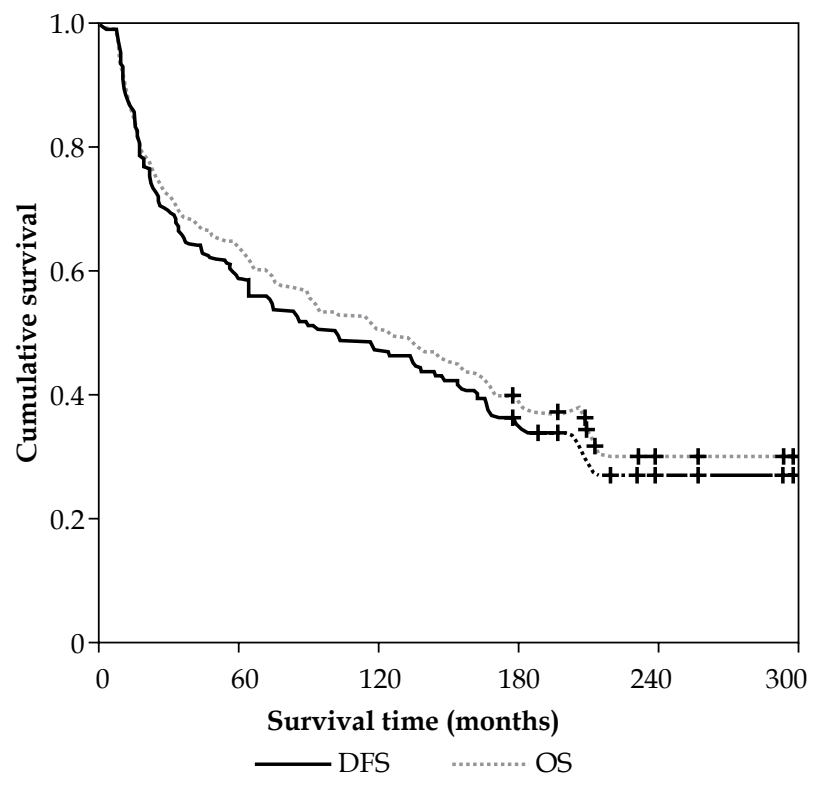

DFS - disease-free survival, OS - overall survival

Fig. 1. Kaplan-Meier disease-free and overall survival curves of IIB FIGO stage cervical cancer patients treated with EBRT and ${ }^{252} \mathrm{Cf}-\mathrm{ICBT}$ lower in the ${ }^{252} \mathrm{Cf}$ group than in the ${ }^{60} \mathrm{Co}$ group $(p=0.28)$. The relapse pattern in both groups is given in Table 3.

Radiation related complications are shown in Table 4. The adverse effects and radiation complications (cystitis, proctitis II-III grade according to RTOG/EORTC scoring and hydronephrosis) were similar in both groups ranging from $1.6 \%$ to $13.5 \%$.

During follow up, the percentage of a second primary cancer in the ${ }^{252} \mathrm{Cf}$ group was similar to the ${ }^{60} \mathrm{Co}$ group: $9.1 \%$ versus $8.1 \%$, respectively $(p=0.79)$.

The results of univariate analysis showed that the histopathologic grade, recurrence, concurrent disease, hemoglobin level, and body mass index were factors that statistically significantly influenced patient prognosis in the ${ }^{252} \mathrm{Cf}$ group $(p<0.05)$; and histopathology, recurrence and metastases were factors that influenced statistically significantly patient prognosis in the ${ }^{60}$ Co group $(p<0.05)$. However, the Cox multiple regression analysis showed that just a histopathologic grade was an independently significant prognostic factor in the ${ }^{252} \mathrm{Cf}$ group, and for recurrence in the ${ }^{60} \mathrm{Co}$ group.

\section{Discussion}

Since the early 1970s, cervical cancer has been recognized as an ideal condition for the use of ${ }^{252} \mathrm{Cf}$ [3]. Several researchers published their experience using ${ }^{252} \mathrm{Cf}$ in cervical cancer treatment with favorable results $[5,6,7,8,9]$. However, to our knowledge, this is the first study, which has revealed the results of curative effects and complications for a period of 20 years for IIB FIGO stage cervical cancer patients treated with ${ }^{252} \mathrm{Cf}$ neutron or ${ }^{60} \mathrm{Co}$ gamma intracavitary brachytherapy combined with external photon beam radiotherapy.

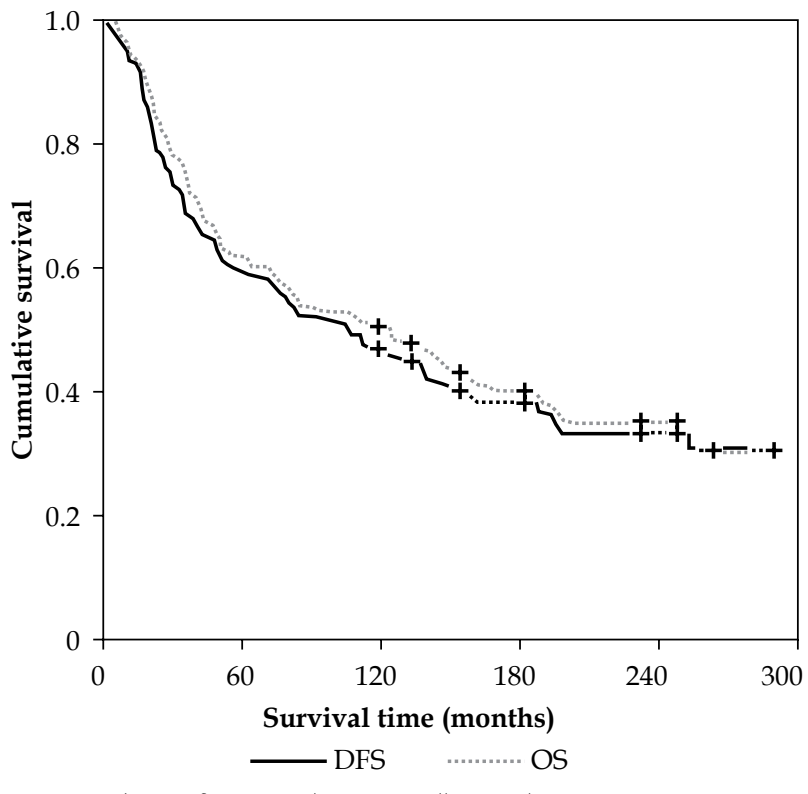

DFS - disease-free survival, OS - overall survival

Fig. 2. Kaplan-Meier disease-free and overall survival curves of IIB FIGO stage cervical cancer patients treated with EBRT and ${ }^{60} \mathrm{Co}-\mathrm{ICBT}$ 
Table 3. Relapse pattern of IIB FIGO stage cervical cancer patients

\begin{tabular}{lccccc} 
& \multicolumn{2}{c}{ EBRT and ${ }^{252}$ Cf-ICBT } & \multicolumn{2}{c}{ EBRT and ${ }^{60}$ Co-ICBT } & $p$-value \\
\cline { 2 - 4 } & Patients & $\%$ & Patients & $\%$ & \\
\hline Recurrence & 112 & 92.6 & 92 & 82.9 & 0.02 \\
\hline No & 9 & 7.4 & 19 & 17.1 & \\
\hline Yes & 111 & & & 87.4 & 0.28
\end{tabular}

EBRT - external-beam radiotherapy, ${ }^{252} \mathrm{Cf}$ - californium-252, ICBT - intracavitary brachytherapy, ${ }^{60} \mathrm{Co}$ - cobalt-60

Table 4. Radiation complications of IIB FIGO stage cervical cancer patients

\begin{tabular}{|c|c|c|c|c|c|}
\hline \multirow[t]{2}{*}{ Complications } & \multicolumn{2}{|c|}{ EBRT and ${ }^{252} \mathrm{Cf}-\mathrm{ICBT}$} & \multicolumn{2}{|c|}{ EBRT and ${ }^{60} \mathrm{Co}-\mathrm{ICBT}$} & \multirow[t]{2}{*}{$p$-value } \\
\hline & Patients & $\%$ & Patients & $\%$ & \\
\hline \multicolumn{6}{|l|}{ Adverse effects } \\
\hline No & 108 & 89.3 & 96 & 86.5 & 0.52 \\
\hline Yes & 13 & 10.7 & 15 & 13.5 & \\
\hline \multicolumn{6}{|l|}{ Cystitis } \\
\hline No & 112 & 92.6 & 103 & 92.8 & 0.95 \\
\hline Yes & 9 & 7.4 & 8 & 7.2 & \\
\hline \multicolumn{6}{|l|}{ Proctitis } \\
\hline No & 119 & 98.4 & 108 & 97.3 & 0.58 \\
\hline Yes & 2 & 1.6 & 3 & 2.7 & \\
\hline \multicolumn{6}{|l|}{ Hydronephrosis } \\
\hline No & 118 & 97.5 & 105 & 94.6 & 0.25 \\
\hline Yes & 3 & 2.5 & 6 & 5.4 & \\
\hline
\end{tabular}

EBRT - external-beam radiotherapy, ${ }^{252} \mathrm{Cf}$ - californium-252, ICBT - intracavitary brachytherapy, ${ }^{60} \mathrm{Co}$ - cobalt-60

The majority of the clinical studies were based on Maruyama's empirical findings in an early application of the ${ }^{252} \mathrm{Cf}$ source in the initial phase of radiotherapy. Maruyama et al. analyzed 41 cases of cervical carcinoma at stage IIB. Patients that received application of the ${ }^{252} \mathrm{Cf}$ implants in the delayed phase of radiotherapy had a significantly greater rate $(40 \%$ vs. $3 \%)$ for developing severe complications (pelvic necrosis, fistulas). Neutron brachytherapy caused tumors to regress rapidly and completely, which allowed the neutron dose to adjacent radiosensitive organs (bladder, rectum, sigmoid colon, and bowel) to become excessive. The delayed ${ }^{252} \mathrm{Cf}$ implant apparently contributed to the greater risk for normal tissue complications [9]. Despite the fact that in our study highdose rate brachytherapy component of ${ }^{252} \mathrm{Cf}$ was added in the $3^{\text {rd }}$ week of EBRT and administered once per week with a fraction dose of $8.0 \mathrm{~Gy} / \mathrm{Gy}_{\text {eq }}$ (point $\mathrm{A}$ ), 5 fractions, our observed-results of radiation complications in ${ }^{252} \mathrm{Cf}$ group are similar comparing with other researchers. Xin Lei et al. also used the HDR brachytherapy component of ${ }^{252} \mathrm{Cf}$ and reported incidence rates of late complications $7.1 \%, 6.2 \%, 4.9 \%$ in the rectum, bladder, and small bowel, respectively [7]. Worse results were reported by Ferrigno et al.: the 5-year incidence rates of late complications in the rectum, bladder, and small bowel were $16.0 \%, 11.0 \%$, and $14.0 \%$, respectively in patients treated with highdose-rate ${ }^{192}$ Ir ICBT [10]. In the Zhao et al. study, early radiation complications such as radiation proctitis $(7.8 \%)$ and radiation cystitis $(4.7 \%)$, and late radiation complication - protracted radiation proctitis - with an incidence of $5.5 \%$ were observed in patients treated with high-doserate ${ }^{252}$ Cf ICBT [6]. Tacev et al. published a randomized study showing that treatment of cervical carcinoma with low-dose-rate ${ }^{252} \mathrm{Cf}$ neutron intraluminal brachytherapy resulted in radiation cystitis, radiation proctitis, and rectal ulcer rates of $16.2 \%, 18.0 \%$ and $0.8 \%$, respectively [8]. 
Considering all this data, the incidence of late radiation proctitis $(1.7 \%)$ was lower and the incidence of late radiation cystitis $(7.4 \%)$ was similar within our study in the ${ }^{252}$ Cf group.

After the treatment, we observed a statistically significant $(p=0.02)$ lower recurrence rate in the ${ }^{252} \mathrm{Cf}$ group $\left({ }^{252} \mathrm{Cf}\right.$ group $-7.4 \%$ vs. $17.1{ }^{60} \mathrm{Co}$ group). The rate of metastases was also lower in the ${ }^{252} \mathrm{Cf}$ group $\left({ }^{252} \mathrm{Cf}\right.$ group $-8.3 \%$ vs. $12.6 \%{ }^{60}$ Co group), but statistically not significant $(p=0.28)$. A randomized phase III trial conducted by Tacev et al., also reported a smaller recurrence rate in the small pelvis in IIB stage cervical cancer patients treated with ${ }^{252} \mathrm{Cf}$, than only treated with gamma irradiation: $7.3 \%$ vs. $16.0 \%$, respectively but not statistically significant. The appearance of distant metastases also was lower in the ${ }^{252} \mathrm{Cf}$ group $\left({ }^{252} \mathrm{Cf}\right.$ group $-5.4 \%$ vs. $12.0 \%$ gamma radiation group). It's important to emphasize that in the Tacev et al. trial, the ${ }^{252} \mathrm{Cf}$ sources were a low dose rate, and the irradiation time lasted several days. This means that the radiobiological ${ }^{252} \mathrm{Cf}$ irradiation was very different in comparison with the HDR ${ }^{252} \mathrm{Cf}$ irradiation [8]. Maruyama et al. also showed a similar recurrence rate $(6.0 \%)$ in II stage (IIA and IIB stages) cervical cancer patients treated with ${ }^{252} \mathrm{Cf}$ but the rate of metastases $(15.0 \%)$ was higher than in our study [5].

The study comparing the ${ }^{252} \mathrm{Cf}$ brachytherapy component and conventional gamma radiation conducted by Tacev et al., showed better OS rates: the overall 5-year survival rate for IIB was better by $13.4 \%$ for ${ }^{252} \mathrm{Cf}$ patients than for patients receiving conventional treatment $(85.4 \%$ vs. $72.0 \%$, respectively). However, we cannot omit the fact that they used low-dose rate brachytherapy [8]. Another report dealing with long-term results with HDR ${ }^{252} \mathrm{Cf}$ treatment combined with EBRT in cervical cancer was published in China by Lei et al., and it showed the overall survival rate for IIB stage at 3 and 5 years was $76.2 \%$ and $66.1 \%$, respectively [7]. Maruyama et al., in whose study the ${ }^{252} \mathrm{Cf}$ neutron brachytherapy was combined with surgery and EBRT, showed similar results to our long-term results. The survival rates for stage II (IIA and IIB) were $62.0 \%$ at 5 years and $61.0 \%$ at 10 years [5]. The 10 years OS results in our study maybe were lower because of the fact that we did not have a surgery component in our study and we analyzed just the IIB stage. However, there is no data for a period of 20 years OS in order to make a comparison.

The risk of developing a second primary cancer after radiotherapy becomes more significant because of the improvement in long-term survival. Xin Lei, in his study, detected just $1.6 \%$ second primary cancers in patients treated with $\mathrm{HDR}{ }^{252} \mathrm{Cf}$ brachytherapy. However, the median of the follow up in the Xin Lei study was short - just 44 months [7]. The other study comparing breast cancer patients undergoing hypofractionated EBRT and brachytherapy by ${ }^{252} \mathrm{Cf}$ sources, with those irradiated by giving conventional EBRT, conducted by Valuckas et al., showed a similar rate of second primary cancers among the groups: conventional radiotherapy $-18.0 \%$ and hypofractionated radiotherapy + postoperative brachytherapy by ${ }^{252} \mathrm{Cf}$ sources $-18.8 \%$ [11]. During our long follow-up, the rate of second primary cancer between the groups was similar. The deep analysis of a second primary cancer after radiotherapy including $\mathrm{HDR}{ }^{252} \mathrm{Cf}$ brachytherapy for cervical cancer was published by Samerdokiene et al., this study shows no significant difference in rates or distribution of second primary cancer in women treated with neutron brachytherapy compared with photon brachytherapy [12]. During our long follow-up, the rate of second primary cancer between the groups was similar: $9.1 \%$ and $8.1 \%$ cases for ${ }^{252} \mathrm{Cf}$ and ${ }^{60} \mathrm{Co}$ groups, respectively.

\section{Conclusions}

Due to the Rjabukin [4] formulae of an equivalent dose calculation and fractionation that was used, our results show similar survival results for ${ }^{252} \mathrm{Cf}$ and ${ }^{60} \mathrm{Co}$ brachytherapy in the treatment of FIGO IIB stage cervix carcinoma patients. The number of tumor recurrences was significant lower in the ${ }^{252} \mathrm{Cf}$ group. The amount of second primary cancers was similar in both groups.

\section{Acknowledgements}

This research was funded by a grant (No MIP-036/ 2013) of the Research Council of Lithuania.

Authors expresses sincere thanks for the statistical calculations to T. Rakovskaja as well to G. Kovács for manuscript editing advice, and to G.P. Elisyutin for excellent technological status of neutron afterloader ANET-V maintained all ten years.

\section{Disclosure}

Authors report no conflict of interest.

\section{References}

1. Ferlay J, Soerjomataram I, Ervik M et al. GLOBOCAN 2012 v1.0, Cancer Incidence and Mortality Worldwide: IARC Cancer Base No. 11. Lyon, France: International Agency for Research on Cancer; 2013. Available from; http://globocan. iarc.fr, accessed on 24/08/2014.

2. Nuclear data for neutron therapy: Status and future needs. IAEA-TECDOC-992, 1997.

3. Zhang M, Xu H, Pan S et al. Low-dose-rate californium-252 neutron intracavitary afterloading radiotherapy combined with conformal radiotherapy for treatment of cervical cancer. Int J Radiat Oncol Biol Phys 2012; 83: 966-971.

4. Ryabukhin YS. Relative biological efficacy of Cf-252 sources. Med Radiol 1985; 30: 53-59 [in Russian].

5. Maruyama Y, van Nagell JR, Yoneda J et al. A review of californium-252 neutron brachytherapy for cervical cancer. Cancer 1991; 68: 1189-1197.

6. Zhao H, Wang K, Sun J et al. Clinical report on external irradiation combined with californium-252 neutron intraliuminal brachytherapy for cervical carcinoma treatment. Tumori 2007; 93: 636-640.

7. Lei X, Qian CY, Qing Y et al. Californium-252 brachytherapy combined with external-beam radiotherapy for cervical cancer: long-term treatment results. Int J Radiat Oncol Biol Phys 2011; 81: 1264-1270.

8. Tacev T, Ptácková B, Strnad V. Californium-252 (252Cf) versus conventional gamma radiation in the brachytherapy of advanced cervical carcinoma long-term treatment results of a randomized study. Strahlenther Onkol 2003; 179: 377-384. 
9. Maruyama Y, van Nagell JR, Yoneda J et al. Schedule in Cf-252 neutron brachytherapy: complications after delayed implant therapy for cervical cancer in a phase II trial. Am J Clin Oncol 1993; 16: 168-174.

10. Ferrigno R, dos Santos Novaes PE, Pellizzon AC et al. High dose-rate brachytherapy in the treatment of uterine cervix cancer. Analysis of dose effectiveness and late complications. Int J Radiat Oncol Biol Phys 2001; 50: 1123-1135.

11. Valuckas KP, Atkocius V, Kuzmickiene I et al. Second malignancies following conventional or combined ${ }^{252} \mathrm{Cf}$ neutron brachytherapy with external beam radiotherapy for breast cancer. J Radiat Res 2013; 54: 872-879.

12. Samerdokiene V, Valuckas KP, Janulionis E et al. Second primary malignancies after radiotherapy including $\mathrm{HDR}{ }^{252} \mathrm{Cf}$ brachytherapy for cervical cancer. Brachytherapy; Available from: http://dx.doi.org/10.1016/j.brachy.2015.06.006, accessed on 12/08/2015. 